\title{
Synthesis of linear D-alt-L peptidomimetics starting from a carbohydrate precursor
}

\author{
Sol C. Parajón Puenzo, Adriana A. Kolender, Sandra E. Martín, Oscar Varela*
}

Dpto. Química Orgánica, CIHIDECAR-CONICET-UBA, Facultad de Ciencias Exactas y Naturales, Buenos

Aires, Argentina - INFIQC, Departamento de Química Orgánica, Facultad de Ciencias Químicas,

Universidad Nacional de Córdoba, Córdoba, Argentina

*e-mailvarela@qo.fcen.uba.ar

Keywords: peptidomimetics, oligopeptides, carbohydrates

\section{INTRODUCTION}

Fundamental building blocks used by nature are amalgamated to produce natural-like, yet unnatural, structural entities with multifunctional groups anchored in a single ensemble. For example, hybrid molecules that maintain the basic structure of a carbohydrate have been obtained. This hybrids include amino and carboxyl functional groups, characteristic of amino acids. Diverse arrays of peptidic templates have been employed for the construction of homo- and heterooligomers that behave as peptidomimetics. These molecules are sometimes able to associate themselves spontaneously (self-assembly process) to form complex architectures. ${ }^{1}$ The novel materials find useful applications as microelectronics, drug delivery and tissue engineering. The linear oligopeptides are also precursor of cyclic peptoids (carbopeptoids) useful as molecular receptors. ${ }^{2}$ We describe herein the synthesis of an amino acid building block starting from inexpensive D-Glucono-1,5-lactone. As the amino containing stereocenter possesses the $S$ configuration, the molecule is combined with $D$ alanine ( $R$ configuration) to give $\mathrm{D}$-alt-L peptides to favor self-assembly processes.

\section{RESULTS AND DISCUSSION}

Treatment of D-Glucono-1,5-lactone (1) with 2,2dimethoxypropane led to conversion into the methyl ester and simultaneous protection as the 3,4:5,6-di$O$-isopropylidene derivative (2). The free hydroxyl group was substituted by azide to obtain 4 , via the triflate 3. Treatment of $\mathbf{4}$ with bis(tribultyltin) oxide $\left[\left(\mathrm{Bu}_{3} \mathrm{Sn}\right)_{2} \mathrm{O}\right]$ gave the free carboxylic acid 5, without racemization or elimination at $\mathrm{C}-2$, as happened with other bases. Coupling of 5 with D-alanine in the presence of 1-hydroxybenzotriazole (HOBt), N,Ndiisopropylethylamine (DIPEA) and a carbodiimide (EDCI) afforded the dipeptide 6. This key intermediate was used as precursor of both the acid 7 (by reaction with $\left.\left(\mathrm{Bu}_{3} \mathrm{Sn}\right)_{2} \mathrm{O}\right)$ and the amine 8 (under Staudinger conditions). Condensation of 7 and $\mathbf{8}$ gave the D-alt-L-tetrapeptide $\mathbf{9}$.
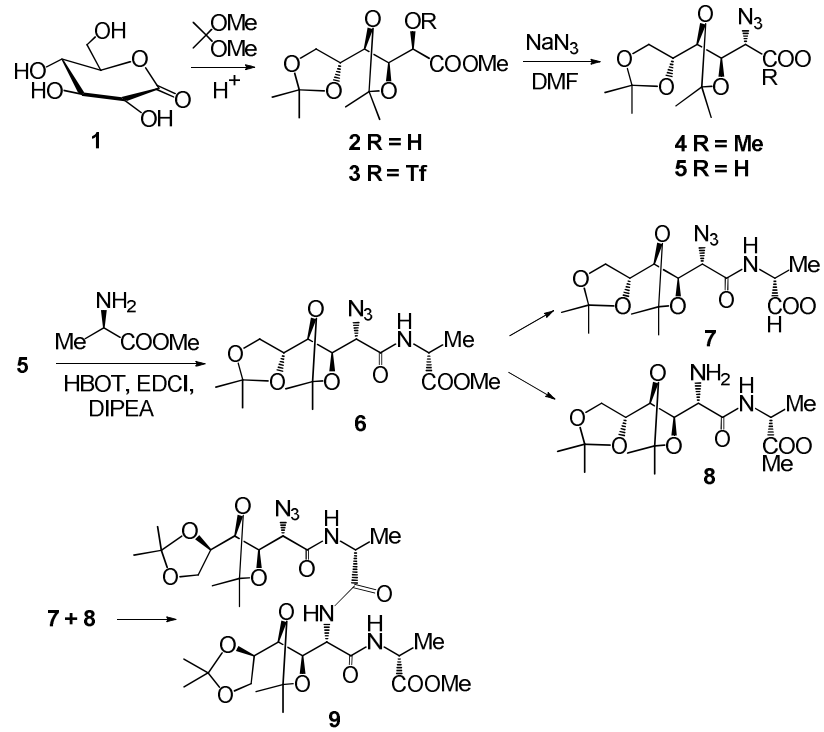

Scheme 1. Synthesis of oligopeptides 6-10.

\section{CONCLUSION}

The sugar-based amino acid precursor 5 has been synthesized straightforward from D-Glucono-1,5lactone. This building block was employed as key intermediate in the stereocontrolled synthesis of linear D-alt-L oligopeptides.

\section{ACKNOWLEDGEMENTS}

We are indebted to ANPCyT, UBA, UNC and CONICET for financial support.

\section{REFERENCES}

${ }^{1}$ Börner, J. C. Prog. Polym. Sci. 2009, 34, 811.

2 Driggers, E. M.; Hale, S. P.; Lee, J.; Terrett, N. K. Nat. Rev. Drug Discovery 2008, 7, 608 .

$14^{\text {th }}$ Brazilian Meeting on Organic Synthesis $-14^{\text {th }}$ BMOS - September 01-05, 2011-Brasilia, Brazil 